\title{
Effect of Debridement on Posttraumatic Soft Tissue Coverage in University of Calabar Teaching Hospital, Nigeria
}

\author{
Ozinko M.O. ${ }^{1}$, Otei, O.O. ${ }^{2}$, Isiwele, E. ${ }^{3}$, Urom, S. E. ${ }^{4}$ \\ ${ }^{1,2,3}$ Burns and Plastic Surgery Unit, Department of Surgery, University of Calabar Teaching Hospital, Calabar, Nigeria \\ ${ }^{4}$ Department of Orthopaedics and Trauma, University of Calabar Teaching Hospital, Calabar, Nigeria
}

\begin{abstract}
Posttraumatic injuries are a common occurrence in our Accident and Emergency department arising from different mechanisms of injuries. Surgical debridement is routinely done as a part of our management protocol. Surgical debridement is done to reduce or prevent wound infection, decrease flap loss, reduce posttraumatic osteomyelitis as well as decrease hospitalization time and management cost. We aim at highlighting the rate of flap viability and infection in relation to the timing of surgical debridement and wound coverage. A retrospective study with the information gathered from the patient case-notes and Casualty Register. All posttraumatic patients that presented between January 2013 and December 2014 were recruited into the study. The patients were resuscitated and wounds debrided within 48hours of presentation.wounds lasting longer than seven days prior to the presentation were excluded from the study on account of late presentation .wound swabs for microscopy, culture, and sensitivity were done. Out of 342 patients seen in the hospital with 568 posttraumatic injuries, 133patients with 205 open wounds were recruited into the study. The malefemale ratio was 1:3:1. Superficial soft tissue infection occurred in 12\% of those with immediate debridement group of 0-24 hours posttrauma while 15.2\% in early debridement between day 2 and 7 post injury period and deep infection was $8.3 \%$. We had $3.6 \%$ flap loss in the immediate wound closure group while $1.2 \%$ in early wound coverage. Surgical debridement is an important procedure in the management of acute traumatic wounds. Adequate early wound debridement and commencement of antibiotics are very important to reduce wound infection and improve flap viability. The use of flaps within the first two days of injury is fraught with the complication of flap loss, thus, 3 -21 days after trauma could be a preferable time for early flap cover.
\end{abstract}

Keywords: Debridement, posttraumatic injuries, soft tissue coverage

\section{Introduction}

Post-traumatic injuries are a common occurrence in our hospital. Accident and Emergency Department is frequently the presenting location for acute wounds which is appropriate given its resources, convenience, and expertise. Posttraumatic injuries are often precipitated by road traffic accidents, falls, assaults, industrial and domestic accidents, bites and sports. These injuries are often presented as lacerations, abrasions, degloving and avulsion injuries, crush or mangled extremities. As the historical and/clinical features surrounding the cutaneous injury process differ, wounds must be evaluated and treated individually [1]. Without proper debridement and wound care, these traumatic wounds can lead to complications such as poor wound healing, infection, and flap loss.

Optimizing wound healing through proper posttraumatic wound management involves debridement and exploration for underlying injuries, control of bacterial burden and appropriate closure. This could call for the adoption of an acceptable protocol by different institutions.

\section{Methodology}

All information was gathered from the accident and emergency register and patient folders. Patients who had posttraumatic injuries presenting to our hospital between January 2013 and 2014 were recruited into the study. A simple protocol that was adopted included: consultation to the plastic and orthopaedic surgeons; the management of the patients with the ATLS protocol; the wounds were washed in accident and emergency department; photographed, and covered with clean dressing; one gram of ceftriaxone and $500 \mathrm{mg}$ of metronidazole were given intravenously; Antitetanus prophylaxis, if indicated and surgical wound debridement and initial or definitive fracture stabilization was carried out within 48 hours of the time of the injury .

In many patients referred to us, we observed inadequate debridement and inappropriate management of traumatic flaps from degloving and avulsion injuries. All the burn patients and those who presented after 7 days were excluded from the study due to late presentation. Co-morbid conditions which could affect wound healing process or infection were also noted. The procedure of posttraumatic wound debridement could be simple or complex depending on the type of wound, location, and function of the involved structures.

In a small wound, an excision of the devitalized tissue, removal of foreign bodies and the irrigation to reduce the bioburden could be done without a tourniquet. Conversely, in large wounds, it is challenging to adopt this method of debridement without a tourniquet. The defects with uneven surface and pockets in the soft tissue bleeding may hide them. In these wounds, blood soon covers the operating field hiding the necrotic areas and deeper extensions of the wound. It becomes impossible to see the typical appearance of the damaged tissues if the operating field is covered with blood. In large wounds, also the blood loss in such a debridement soon reaches an amount significant enough to need replacement. Therefore, we start debridement in a 


\section{International Journal of Science and Research (IJSR) \\ ISSN (Online): 2319-7064 \\ Index Copernicus Value (2013): 6.14 | Impact Factor (2015): 6.391}

bloodless field with a tourniquet. This enables us to observe the appearance of the tissue, to find and eliminate thoroughly the foreign body in the wound, and to perform accurate haemostasis of the vessels in the excision, thus saving blood loss .After the excision is done, the tourniquet is released and at this stage also we can assess the quality of the excision by the bleeding of the excised surfaces. Debridement in a bloodless field is far more accurate and because of the low blood loss is less traumatizing to the patient.

It is relatively easy to differentiate damaged tissues in a bloodless field. The appearance and consistency of the tissues are the main guidelines to be followed. Avulsed skin and muscles have to be excised; tendons which are not crushed are cleaned. They may be sutured or tagged for later reconstruction. Exposed bone is thoroughly cleaned and free fragments may be used as bone grafts to bridge defects. Injured vessels are ligated if they are not essential. The nerves are the only structures for which debridement is not radical for the purpose of easy apposition. Their free ends should be sutured to the surrounding tissue to prevent retraction of the nerve and to mark the stump for secondary reconstruction. Primary grafting of the nerve can be performed. This is advisable in clean wounds and shorter nerve defects. The wound is thoroughly irrigated with copious amount of fluid. The fluid of choice is normal saline. The amount of the irrigating fluid depends on the extent of the wound. Also, serial debridement is done for large traumatic wounds such as degloving or avulsion injuries and mangled extremity.

In minor lacerations, they could be sutured immediately while the large ones could be reconstructed immediately or delayed. Avulsion and degloving injuries could be covered when tissue demarcation and edema have subsided. Locoregional and distant flaps were used. Muscle flaps were used to cover exposed bones. No free flap was used

Monitoring of wounds for flap viability and infection were done using both clinical and laboratory tests such as wound swab for microscopy, culture and sensitivity and temperature. The limbs were elevated to reduce edema. Antibiotics were given initially empirically and later therapeutically based on wound swab culture and sensitivity.

\section{Result}

Out of 342 patients seen in the hospital with 568 posttraumatic injuries, 133 patients with 205 open wounds were recruited into the study. The male- female ratio was 1.3: 1. Superficial soft tissue infection occurred in $12 \%$ of those with immediate debridement group of $0-24$ hours post-trauma while $15.2 \%$ in early debridement between day $2-7$ post-injury period and deep wound infection was $8.3 \%$. We had $3.6 \%$ flap loss in the immediate wound closure group while $1.2 \%$ in early wound closure group.

\section{Discussion}

This was a retrospective audit that was intended to highlight the effect of debridement on soft tissue cover of posttraumatic wounds. This was aimed at looking at the rate of flap viability and infection in relation to the timing of surgical debridement and wound cover.

Surgical debridement is the procedure of removing all devitalized tissues, foreign bodies and reducing the wound bioburden by sharp excision and copious irrigation. The period for debridement was divided into an immediate period from 0-24hours and from day $2-7$ as early debridement. Post-traumatic wounds are seen on day 8 and above were excluded on the basis of late presentation with significant wound infection. We also had some large complex wounds serially debrided on account of inadequate wound debridement as the devitalized tissues become clearly demarcated subsequently.

The primary goals for traumatic wound care are to attain a functional closure, decrease potential risk for infection and minimizing scar formation [2]. As such, a complete patient history is needed to determine the outcome risk for and type of potential contamination, activity at the time of injury, functional changes and any co-morbid illness that may prevent or decrease normal healing. Injury to and through the epidermis can allow for bacterial migration and subsequent inflammation and infection if not appropriately managed. Associated risk factors for wound infection are increased age, diabetes mellitus, complex wounds, wound contamination, or the presence of foreign body [3]. In addition, tetanus prophylaxis and allergy history should be obtained prior to initiation of wound management. The time from injury to acute wound management is an essential consideration for appropriate wound closure.

The wound in which soil or dirt are embedded are considered contaminated at the time of injury and may need to be left opened if adequate debridement is uncertain or may be required in more aggressive operative procedures.

The physical examination of a wound requires assessment of location, extent, type of tissue involved, neurovascular status and associated contaminants. It is imperative to assess all adjoining joints through a full range of motion paying particular attention to the position at the time of initial assessment.

The simple management protocol that was used in the method has reduced the incidence of wound infection and increased flap survival. Debridement was done within 48 hours of presentation but all the patients were not debrided within this time frame because of hospital logistics.

Current guidelines supported by the British orthopaedic Association (BOA) suggest that the first surgical procedure should be performed within 6 hours of the original accident [4].The rationale for BOA's Six- Hour Rule is believed to originate from Freidrich's historical study of guinea pigs [5]. In 1898, he evaluated the replication of bacteria and deemed 6hours to be a critical limit after which massive replication was detected.

The threshold for immediate debridement is the 6hour interval from the original accident, that is, the six- hour rule. Gordina [6] introduced three categories of wound closure namely; immediate, early and delayed. Immediate was 


\section{International Journal of Science and Research (IJSR) \\ ISSN (Online): 2319-7064 \\ Index Copernicus Value (2013): 6.14 | Impact Factor (2015): 6.391}

closure within 72 hours, early was between 72 hours and 3 months and delayed was beyond 3months. Gopal et al [7] simply used 72 hour figure, and categorized anything within this time scale as early and anything beyond it as delayed.

Ostermann et al [8] defined delayed as the wound closed after 5 days and this was also adopted by the British orthopaedic Association and the British Association of Plastic Surgeons guidelines[4].Therefore taking all these studies into consideration they defined immediate closure as one that has been carried out at the first surgical procedure within 72 hours . Early closure is carried within the period of 3 to 5 days and any closure beyond 5 days is delayed closure

In 2004, Spencer et al [9] published a 5- year prospective audit from a District General Hospital looking directly into the time delay in open fractures. The study had an overall infection rate of $10.4 \%$ having achieved the 6hour goal in $60 \%$ of cases. They found no statistically significant evidence to support the 6hour theory.

In another study, Gopal et al [7] published a retrospective analysis of 84 patients who had suffered a Gustilo $3 \mathrm{~b}$ or $3 \mathrm{c}$ fracture of the tibia. All had been treated by a combined orthopaedic and plastic surgery services with an aggressive protocol of extensive debridement, skeletal stabilization and early soft tissue cover with vascularised muscle flap. These approaches led to only four amputations. The superficial infection rate overall was $9 \%$ with a deep infection at the fracture site of $3 \%$. The authors recommended immediate transfer to a specialized center with a plastic surgeon on site, immediate internal fixation, and soft tissue cover. If it is not possible to transfer the patient, then they recommended immediate debridement and external fixation on prior to transfer.

In our study, we considered the use of antibiotic therapy in posttraumatic wound management as part of our protocol. Although 6hours is considered the golden time between injury and risk for infection, Edlich et al [10] recommended 3hours to be a more conservative interval for the commencement of antibiotic therapy when appropriate.

A controlled study by Ichikawa [11] found baseline wound infection rates that varied by degree of contamination; $2.6 \%$ in clean-contaminated, $5.8 \%$ in contaminated and $20.8 \%$ in dirty wounds. In a study of 1,142 acute wounds, Lammers [12] found an overall infection rate of $7.2 \%$, although rates vary based on different locations of the body with scalp wounds having an infection rate of $1.7 \%$ whereas the thigh and leg wounds had infection rate as high as $23 \%$. The study also pointed out that the most predictive factors for acute wound infection were wound location, wound age, depth, configuration, contamination and patient's age.

In this study, superficial surgical site infection occurred in $12 \%$ in the immediate debridement group of 0 -24hours while $15.2 \%$ in early wound debridement between day $2-7$ post-injury period. The microbial burdens were similar to previous study in the hospital [13].
Considering the relevance of antibiotics and debridement in wound infection, Wilkins [14] suggested that the single most important factor for reducing the infection rate is the early administration of antibiotics, though Gustilo [15] stated that early debridement is the single most important factor in obtaining good results in an open fracture. However, Merrit [16] concluded that the combination of antibiotics and the adequacy of the debridement are more important than the timing of the debridement.

The study had $3.6 \%$ flap loss in immediate wound closure (which is within the first 48hours of injury) while $1.2 \%$ in early wound closure (which is between 3 and 21 days of injury). Gustilo [7] had a similar result and, therefore, advocated early debridement and immediate wound closure which is between the $3^{\text {rd }}$ day and the $3^{\text {rd }}$ month of injury.

\section{Conclusion}

Surgical debridement is an important procedure for acute wound management. Adequate early wound debridement and commencement of antibiotics are the two most important factors to reduce wound infection and increase flap viability. The use of flaps within the first two days of injury is fraught with the complication of flap loss, thus, 3 21 days after trauma could be a preferable time for early flap cover.

\section{References}

[1] Wicks, B.A., Ayello, E.A., Sibbald, R.G. Acute wound management: Revisiting the approach to assessment, irrigation and closure considerations. International Journal of Emergency Medicine. 2010; 3 (4):399-407.

[2] Percival, N.J .Classification of wounds and their management. Journal of Surgery. 2002; 20 (5):114-117.

[3] Hollander, J. E., Singer, A.J., Valentine, S.M., Shoffer, F.S. Risk factors for infection in Patients with traumatic lacerations. Academic Emergency. Medicine. 2001; 8 (7):716-720.

[4] British Orthopaedic Association and British Association of Plastic Surgeons. A Working Party Report: The Management of open tibial fractures. 1997. http.// www.boa.uk.

[5] American College of Surgeons. Advanced Trauma Life Support Student Course Manual $7^{\text {th }}$ Edition.2002.

[6] Godina , M . Early Microsurgical Reconstruction of Complex Trauma of the Extremities. Plastic Surgery. 1986;78:285-292

[7] Gopal, S., Majumder S., Batchelor, A.G. Fix and Flap: The radical orthopaedic and Plastic treatment of severe open fractures of the tibia. The journal of Bone \& Joint Surgery. 2000; 82:959-966

[8] Ostermann, P.A, Henry, S.L, Seligson, D. Timing of wound closure in severe compound fractures. Orthopaedics 1994; 17:397-399

[9] Spencer, J. Smith, A., Woods, D. The effect of time delay on infection in open long bone fractures: A 5year prospective audit from a district General Hospital. Annals of Royal College of Surgeons England. 2004; 86:108-112 


\section{International Journal of Science and Research (IJSR) \\ ISSN (Online): 2319-7064}

Index Copernicus Value (2013): 6.14 | Impact Factor (2015): 6.391

[10]Edlich, R.F, Madden, J.E, Prusak , M, Panek , O.H. Studies in the Management of the contaminated wound. American Journal of Surgery. 1971; 1284-1302.

[11] Ichikawa IM, Okazaki T.A Prospective Study of Antibiotic Proctocols for managing surgical Site Infection in Children. Journal of Pediatric Surgery. 2007;42 (6):1002-1007

[12]Lammers, R.L., Hudson, D.L., and Seaman, M.E. Prediction of traumatic wound infection with a neural network - derived decision model. American Journal of Emergency Medicine. 2003; 20 (1):1-7

[13] Ozinko, M.O., Otei, O.O., Ekpo, R. G., and Ofutet, E.O. Honey Dressing in the management of malodorous wounds in Calabar, Southern Nigeria. International Journal of Science and Research. 2015; 4(9):831-833.

[14]Patzakis, M.J., and Wilkins, J. Factors influencing infection rate in open fracture wounds. Clinical Orthopaedics and Related Research. 1988; 36-40.

[15] Gustilo, R.B. and Anderson, J .T. Prevention of infection in the treatment of 1025 open fractures of long bones: Retrospective analyses. Journal of Bone \& Joint Surgery. 1976; 58:453-458

[16] Meritt, k. Factors increasing the risk of infection in patients with open fractures. Jorunal of Trauma. 1988; 28:823-827 